\title{
Relationship Between Multidrug-Resistant Enterobacterales and Obesity in Older Adults
}

\author{
Navaneeth Narayanan $\mathbb{I}^{1,2}$ \\ Tiffany Lin' \\ David Vinarov ${ }^{3}$ \\ Thomas Bucek ${ }^{3}$ \\ Liya Johnson' \\ Cheryl Mathew' \\ Saira Chaudhry ${ }^{4}$ \\ Luigi Brunetti (D) ${ }^{1,5}$ \\ 'Department of Pharmacy Practice, \\ Rutgers University, Ernest Mario School \\ of Pharmacy, Piscataway, NJ, USA; \\ ${ }^{2}$ Division of Infectious Diseases, Rutgers \\ University, Robert Wood Johnson \\ Medical School, New Brunswick, NJ, \\ USA; ${ }^{3}$ Rutgers, The State University of \\ New Jersey, New Brunswick, NJ, USA; \\ ${ }^{4}$ Merck Research Laboratories, \\ Kenilworth, NJ, USA; ${ }^{5}$ Center of \\ Excellence in Pharmaceutical \\ Translational Research and Education, \\ Ernest Mario School of Pharmacy, \\ Piscataway, NJ, USA
}

\begin{abstract}
Purpose: The objective of our study was to determine if obesity is associated with the presence of multidrug-resistant (MDR) bacteria among Enterobacterales.

Patients and Methods: This two-center cohort study included adult hospitalized patients with at least one specimen sampled from any site for bacterial culture yielding an Enterobacterales bacterial species from November 2016 to May 2017. Study groups were stratified by obesity status based on body mass index $<30 \mathrm{~kg} / \mathrm{m}^{2}$ (non-obese) and $\geq 30 \mathrm{~kg} / \mathrm{m}^{2}$ (obese). The primary outcome was the presence of gram-negative MDR bacteria defined as presumptive extendedspectrum beta-lactamase (ESBL)-producing Enterobacterales (ceftriaxone resistance) or carbapenem-resistant Enterobacterales (CRE). A multivariable logistic regression model was fit to estimate the adjusted odds ratio while controlling for potential confounders.

Results: A total of 366 patients, 238 non-obese and 128 obese, were included. The most common gram-negative bacterial species identified was Escherichia coli (64.2\%). There was a higher proportion of gram-negative MDR bacteria in obese versus non-obese patients (18.8 versus $11.3 \%, P=0.057)$. Obesity was independently associated with gram-negative MDR bacteria after controlling for confounders (adjusted odds ratio, 1.92; 95\% CI 1.03-3.60). The association did not significantly vary by diabetes status (interaction term $P=0.792$ ).

Conclusion: Among older adult hospitalized patients, obesity was independently associated with the presence of a gram-negative MDR bacteria (presumptive ESBL or CRE) in a culture.
\end{abstract}

Keywords: antibiotics, resistance, obesity, multi-drug resistance, ESBL, MDR bacteria

\section{Introduction}

Obesity is a significant public health crisis that has reached epidemic proportions. In the United States from 2000 to 2018, the prevalence of obesity (body mass index, BMI $\geq 30 \mathrm{~kg} / \mathrm{m}^{2}$ ) and severe obesity (BMI $\geq 40 \mathrm{~kg} / \mathrm{m}^{2}$ ) grew from $30.5 \%$ to $42.4 \%$ and $4.7 \%$ to $9.2 \%$, respectively, in adults aged 20 and over. ${ }^{1}$ This trend is expected to continue nationally, with nearly one in two adults overall projected to be obese and nearly one in four adults projected to be severely obese by 2030 . $^{2,3}$

The increasing incidence of obesity places a heavy burden on healthcare systems. While obesity is a well-known risk factor for many chronic conditions like cardiovascular disease, hypertension, and diabetes, it has also been associated with a higher risk of infection. ${ }^{4,5}$ The underlying mechanism is unclear but potentially linked to dysregulations in inflammation and immunity. ${ }^{5}$ Treatment of infection in individuals with obesity is prone to antibiotic failure, and those hospitalized with infection have increased in-hospital mortality compared to individuals without obesity. $^{5-10}$
Correspondence: Luigi Brunetti 160 Frelinghuysen Road, Piscataway, NJ, 08854, USA

Tel + I 9085952645

Email brunetti@pharmacy.rutgers.edu 
The management of infections in the obese population is complicated by alterations in body composition, with obese patients having a higher proportion of adipose tissue, a lower proportion of total body water, and lower lean body mass. ${ }^{11}$ These changes impact many pharmacokinetic parameters: drug absorption, distribution, metabolism, and excretion. $^{12,13}$ Subsequent variability in drug exposure impacts attainment of pharmacodynamic target concentrations necessary for eradication of infection. One comparative study found an increased frequency of antimicrobial resistance genetic markers in fecal specimens of obese individuals compared to those of eutrophic subjects. ${ }^{14}$ In addition, there is a lack of robust clinical data on the dosing of antibiotics in obese patients, and standard dosing may result in drug underexposure. ${ }^{13}$ Even when antibiotic dosing recommendations do exist, they are not generally followed in practice. ${ }^{15}$

Underdosing antibiotics in individuals with obesity may increase their risk for developing multidrug-resistant (MDR) bacteria. In vitro and in vivo data suggest that inappropriate antibiotic dosing contributes to higher rates of antibiotic resistance. ${ }^{16}$ Of particular concern are the MDR bacteria among gram-negative Enterobacterales because of their pervasive nature, ability to cause a wide range of clinical infections, and rapid promulgation of plasmid-mediated resistance genes to other organisms. ${ }^{17}$ In 2017, the World Health Organization categorized extended-spectrum beta-lactamase (ESBL)-producing and carbapenem-resistant Enterobacterales (CRE) as criticalpriority pathogens, acknowledging their ranking among families of bacteria that pose the greatest threat to global human health. ${ }^{18}$ These dangers are recognized nationally as well; in their 2019 report, the Centers for Disease Control and Prevention categorized CRE as an urgent, or highest level, threat, and ESBL as a serious, or secondhighest level, threat. ${ }^{19}$ With rates of ESBL and CRE infections on a precipitous rise, understanding of risk factors for MDR bacterial infection is of utmost importance. ${ }^{20,21}$ The objective of our study was to evaluate whether obesity is associated with the presence of MDR bacteria among Enterobacterales on culture in hospitalized patients.

\section{Materials and Methods}

\section{Study Design and Population}

We conducted a two-center, retrospective cohort study of adult hospitalized patients with at least one specimen sampled for bacterial culture yielding an Enterobacterales bacterial species from November 2016 to May 2017. This study was undertaken in two urban teaching hospitals in the Northeast United States. All patients aged 18 years or older who had bacterial cultures drawn and their weight and height recorded were eligible for inclusion. Patients were identified by querying the microbiology laboratory database and were further reviewed via electronic medical records. Specimen samples were included irrespective of infection type (ie, community or healthcare-associated) and origin (ie, lung, skin and soft tissue, blood, genitourinary, and intra-abdominal). The respective Institutional Review Board for each study site approved this study (Robert Wood Johnson University Hospital Somerset [IRB17-16] and Rutgers Biomedical and Health Sciences [Pro20170001181]).

\section{Measurements}

The predictor of interest was BMI. Patient weight and height were extracted from the electronic health record. Study groups were stratified by obesity status: BMI $<30 \mathrm{~kg} / \mathrm{m}^{2}$ (non-obese) and $\geq 30 \mathrm{~kg} / \mathrm{m}^{2}$ (obese). Multiple covariates were recorded to determine differences between study groups and control for potential confounding factors. Covariates included basic demographics, history of prior antibiotic therapy in the past 30 days, immunosuppression, transfer from other healthcare facilities, sampling specimens from a critical care unit, and comorbidities as measured by the Charlson-Deyo comorbidity index. Prior antibiotic therapy was determined via prescription fill history in the health record. Immunosuppression was defined as the presence of corticosteroids, cyclosporine, tacrolimus, or other immunosuppressants in a patient's medication list. The Charlson-Deyo comorbidity index was calculated using International Classification of Diseases 9th revision, clinical modification (ICD-9-CM) or International Classification of Diseases 10th revision, clinical modification (ICD-10-CM) codes as a proxy for patient comorbid disease burden.

\section{Outcomes}

The study's primary endpoint was the presence of gramnegative MDR bacteria on a culture defined as presumptive ESBL-producing Enterobacterales (ceftriaxone resistance) or CRE. Phenotypic susceptibility was determined by broth microdilution via automated susceptibility testing platforms at each respective hospital (BD Phoenix or VITEK 2). The breakpoints used to define MDR bacteria are based on those set by the Clinical and Laboratory 
Standard Institute (CLSI). ${ }^{22}$ All cultures performed were clinical cultures.

\section{Statistical Analysis}

Statistical analysis was performed using SAS 9.4 (SAS Institute Inc., Cary, NC). Data for missing covariates were adjudicated with manual chart review. Descriptive statistics were reported for all data. Categorical data were analyzed with chi-square or Fisher exact tests as appropriate. The normality of data was assessed with a visual inspection of histograms and the Shapiro-Wilk test. Continuous data were analyzed with the Student's $t$-test or the Mann-Whitney $U$-test if nonparametric distributions were identified. A multivariable logistic regression model was fit to estimate the adjusted odds ratio (OR) while controlling for potential confounders. Confounders with a relation $(P<0.1)$ to both outcome (gram-negative MDR bacteria) and exposure (obesity status) were considered for inclusion in the adjusted model. A backward selection procedure with a significance level of 0.05 to remain in the model was used to construct the final adjusted model. Only covariates with a priori knowledge suggesting confounding were included in the final regression model selection. The significance level was set at a $P$-value of $<0.05$.

The complex relationship between obesity and diabetes warrants a close examination since various pharmacokinetic and pharmacodynamic changes may be present in each comorbidity alone and in combination. To better disentangle the association of the two metabolic diseases and the presence of gram-negative MDR bacteria, we sought to investigate the potential effect modification by diabetes status. We included the interaction term BMI-bydiabetes in the final multivariable logistic regression model (ie, interaction model) to analyze the presence of interaction.

\section{Results}

A total of 366 patients were included in the analysis, 238 non-obese and 128 obese patients. Baseline characteristics were similar between groups (Table 1). The mean age in the study was 77 years, and $63 \%$ of patients were female. Significantly more patients in the obese group had higher median creatinine clearance $(71.9 \mathrm{~mL} / \mathrm{min}$ versus $48.2 \mathrm{~mL} / \mathrm{min}, P<0.001$ ), a higher proportion of history of cancer $(18 \%$ versus $9.7 \%, P=0.022)$, and a higher incidence of diabetes mellitus $(41.4 \%$ versus $31.1 \%, P=0.048)$ compared with the non-obese group.
The most common culture source was the urine (73.5\%), followed by blood (17.6\%), abscess (5\%), and respiratory (2.5\%) (Table 2). Table 3 provides a summary of gram-negative MDR bacteria from bacterial culture. The most common gram-negative species identified was E. coli $(64.2 \%)$. Among gram-negative MDR bacteria, ESBL were the most commonly isolated, comprising $95.8 \%$ of obese and $92.6 \%$ of non-obese resistant samples.

There was a higher proportion of gram-negative MDR bacteria in obese versus non-obese patients (18.8 versus $11.3 \%, P=0.057)$ in the unadjusted analysis. After adjusting for study hospital and history of cancer in the multivariable logistic regression analysis, obesity was independently associated with 1.92 times higher odds of gram-negative MDR bacteria on culture (adjusted OR, 1.92; $95 \%$ confidence interval, $1.03-3.60, P=0.041)$. The association of obesity and gram-negative MDR bacteria did not significantly vary by diabetes status (interaction term $P=0.792$ ).

\section{Discussion}

Due to complex changes in body composition, obese patients are thought to be frequently underdosed with antibiotics; the resultant inadequate systemic exposure may put them at a higher risk for drug-resistant organisms. Data directly supporting the link between obesity and increased risk of MDR bacteria is lacking. Our study bridges this gap and adds to the body of evidence supporting the increased prevalence of drug-resistant organisms in the obese population. We observed a significant association of obesity status and the presence of gramnegative MDR bacteria on bacterial culture after adjusting for confounding. Of note, the data are derived from an older cohort of adults; therefore, extrapolation to younger patient populations requires confirmation. Additionally, we did not find that diabetes status was a significant effect modifier on this relationship.

Prior studies have observed the relationship between obesity and a higher risk of infection and poorer outcomes related to infection. ${ }^{5-10}$ Our findings provide further evidence on the detrimental impact of obesity on individual and public health related to infectious diseases. Antimicrobial resistance is a global health threat requiring immediate and sustained action. Further clinical and translational studies are necessary to understand the underlying mechanism of this epidemiological finding. 
Table I Baseline Characteristics Stratified by Obesity Status (Total $n=366$ )

\begin{tabular}{|c|c|c|c|}
\hline Characteristics & Non-Obese $(n=238)$ & Obese $(n=128)$ & $P$ value \\
\hline BMI $\left(\mathrm{kg} / \mathrm{m}^{2}\right)$ & $24.8(21.4-27.3)$ & $34.1(32.2-38.4)$ & $<0.001$ \\
\hline Age (years) & $79.3(66-86)$ & $75.1(63.4-84.1)$ & 0.067 \\
\hline Age $>65$ years, $n(\%)$ & $182(76.5)$ & $93(72.7)$ & 0.421 \\
\hline Female, n (\%) & 147 (62) & $82(65.1)$ & 0.566 \\
\hline \multicolumn{4}{|l|}{ Race/ethnicity, n (\%) } \\
\hline White, non-Hispanic & $160(67.2)$ & $94(73.4)$ & 0.219 \\
\hline Black & $23(9.7)$ & $16(12.5)$ & 0.402 \\
\hline Asian & $19(8.0)$ & $4(3.1)$ & 0.068 \\
\hline Hispanic & $19(8.0)$ & $7(5.5)$ & 0.372 \\
\hline Other or unknown & $17(7.1)$ & $7(5.5)$ & 0.537 \\
\hline $\mathrm{SCr}(\mathrm{mg} / \mathrm{dL})$ & I (0.7-I.4) & $\mathrm{I}(0.8-\mid .5)$ & 0.189 \\
\hline $\mathrm{CrCl}(\mathrm{mL} / \mathrm{min})$ & $48.2(34.2-80.5)$ & $71.9(46-117.6)$ & $<0.001$ \\
\hline Length of stay (days) & $2(0-7)$ & I (0-6) & 0.167 \\
\hline Charlson-Deyo index, median (range) & $2(I-4)$ & $2(I-4)$ & 0.180 \\
\hline \multicolumn{4}{|l|}{ Hospital, n (\%) } \\
\hline A & $115(7 \mid .4)$ & $46(28.6)$ & 0.023 \\
\hline B & $123(60)$ & $82(40)$ & 0.023 \\
\hline Culture from ICU, n (\%) & $32(13.5)$ & $21(16.4)$ & 0.443 \\
\hline On hemodialysis, $\mathrm{n}(\%)$ & $4(1.7)$ & $\mathrm{I}(0.8)$ & 0.482 \\
\hline Antibiotic allergy, n (\%) & $50(22)$ & $32(26)$ & 0.400 \\
\hline \multicolumn{4}{|l|}{ History of antibiotics, $\mathrm{n}$ (\%) } \\
\hline Within 30 days & $20(8.7)$ & $13(10.7)$ & 0.541 \\
\hline Greater than 30 days & $43(18.2)$ & $27(21.1)$ & 0.507 \\
\hline \multicolumn{4}{|l|}{ History of corticosteroids, n (\%) } \\
\hline Within 30 days & II (5.4) & $5(4.2)$ & 0.634 \\
\hline Greater than 30 days & $20(9.8)$ & $7(5.9)$ & 0.224 \\
\hline Immunosuppressants, n (\%) & $2(1)$ & $3(2.5)$ & 0.283 \\
\hline Diabetes mellitus, n (\%) & $74(31.1)$ & $53(41.4)$ & 0.048 \\
\hline Transfer from healthcare facility, $\mathrm{n}(\%)$ & $42(17.8)$ & $28(21.9)$ & 0.346 \\
\hline History of cancer, n (\%) & $23(9.7)$ & $23(18)$ & 0.022 \\
\hline
\end{tabular}

Notes: All continuous variables presented as median (interquartile range) and categorical variables presented as $\mathrm{n}$ (\%).

Abbreviations: $\mathrm{BMI}$, body mass index; $\mathrm{SCr}$, serum creatinine; $\mathrm{CrCl}$, creatinine clearance; ICU, intensive care unit.

The influence of the gut microbiota on human health is a rapidly emerging field. Gut dysbiosis has been reported in patients with obesity and increased intestinal permeability may also be present in this patient population. ${ }^{23}$ This postulation is affirmed by studies that report translocation of gut microbiota to extra-intestinal tissue. ${ }^{24,25}$ In particular, plasma and adipose samples from individuals with severe obesity and diabetes displayed similarities to gut associated signatures. Interestingly, and related to our research findings, plasma samples were enriched with Enterobacterales in one human study. ${ }^{24}$ Whether translocation of MDR Enterobacterales from the gut in individuals with obesity poses a risk for resistant infection or body site colonization requires further study.

There are some limitations to this study that should be considered. We only had access to recorded data in the 
Table 2 Comparison of Culture Source and Organism Present on the First Culture Obtained

\begin{tabular}{|l|l|l|l|}
\hline $\begin{array}{l}\text { Sourcel } \\
\text { Organism }\end{array}$ & $\begin{array}{l}\text { Non-Obese, } \\
\text { n (\%) }\end{array}$ & $\begin{array}{l}\text { Obese, } \\
\text { n (\%) }\end{array}$ & P value \\
\hline Urine & $175(73.5)$ & $95(74.2)$ & 0.112 \\
E. coli & $117(66.9)$ & $61(64.2)$ & 0.661 \\
Klebsiella spp. & $24(13.7)$ & $21(22.1)$ & 0.077 \\
Proteus spp. & $22(12.6)$ & $0(0.0)$ & $<0.001$ \\
Other & $12(6.9)$ & $13(13.7)$ & 0.066 \\
\hline Blood & $42(17.6)$ & $21(22.1)$ & 0.764 \\
E. coli & $29(69.0)$ & $12(57.1)$ & 0.350 \\
Klebsiella spp. & $7(16.0)$ & $6(28.6)$ & 0.272 \\
Other & $6(14.3)$ & $3(14.3)$ & $>0.999$ \\
\hline Abscess & $12(5.0)$ & $8(6.3)$ & 0.628 \\
E. coli & $7(58.3)$ & $4(50.0)$ & $>0.999$ \\
Klebsiella spp. & $1(8.3)$ & $2(25.0)$ & 0.686 \\
Other & $4(33.3)$ & $2(25.0)$ & $>0.999$ \\
\hline Respiratory & $6(2.5)$ & $4(4.2)$ & 0.735 \\
E. coli & $2(33.3)$ & $2(50.0)$ & $>0.999$ \\
Klebsiella spp. & $3(50.0)$ & $2(50.0)$ & $>0.999$ \\
Other & $1(16.7)$ & $0(0.0)$ & $>0.999$ \\
\hline
\end{tabular}

Table 3 Unadjusted Gram-Negative MDR Bacteria Rates Stratified by Obesity Status

\begin{tabular}{|l|c|c|c|c|}
\hline Organism & $\begin{array}{c}\text { Overall, } \\
\mathbf{n}(\%)\end{array}$ & $\begin{array}{c}\text { Non-Obese, } \\
\mathbf{n}(\%)\end{array}$ & $\begin{array}{c}\text { Obese, } \\
\mathbf{n}(\%)\end{array}$ & $\boldsymbol{P}$ value \\
\hline $\begin{array}{l}\text { ESBL or CRE on } \\
\text { culture }\end{array}$ & $51(13.9)$ & $27(11.3)$ & $24(18.8)$ & 0.057 \\
\hline ESBL & $48(13.1)$ & $25(10.5)$ & $23(17.9)$ & 0.049 \\
\hline $\begin{array}{l}\text { Enterobacterales } \\
\begin{array}{l}\text { E. coli } \\
\text { Klebsiella spp. } \\
\text { Other }\end{array}\end{array}$ & $235(64.2)$ & $156(65.6)$ & $79(61.7)$ & 0.468 \\
\hline
\end{tabular}

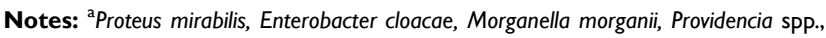
Citrobacter spp.

Abbreviations: MDR, multidrug-resistance; ESBL, extended-spectrum beta-lactamaseproducing Enterobacterales; CRE, carbapenem-resistant Enterobacterales.

patient's medical record and assumed that clinical information was correctly documented. In addition, as with all retrospective studies, confounding and information bias are possible. Many other variables can influence drug resistance, including prior antibiotic use, comorbidities such as diabetes or cancer, immunosuppression, the severity of illness, length of stay, and transfer from another facility. We accounted for these potential confounders in our analysis using multivariable logistic regression. After considering these confounders in our regression model, obesity was independently associated with gram-negative MDR bacteria. The presence of indwelling devices and frequency of hospitalization are additional potential confounders for MDR bacterial infection that were not collected; however, the adjustments as mentioned earlier, such as those for comorbidities and location, serve as a broad proxy. While residual confounding is always a concern with retrospective data, the study did account for major known contributors to antibiotic resistance.

With the worldwide rates of both obesity and antibiotic-resistant organisms on a steep incline, it is imperative to understand the relationship between them. The evidence generated by this study supports the notion that obesity is indeed associated with an increased risk of gram-negative MDR bacteria. This association is clinically relevant with obesity as a potential predictor of antibiotic resistance, establishing obesity-specific antibiotic dosing and therapeutic monitoring guidelines are imperative. These considerations should also be integral when developing new anti-infective medications. Further studies are needed to investigate the causal pathway between obesity and MDR bacteria and elucidate effective interventions to reduce the emergence of drug resistance.

\section{Ethics and Consent}

Patient consent was waived due to the retrospective nature of the study that does not disclose patients' identities. The study was conducted according to the guidelines of the Declaration of Helsinki and approved by the Institutional Review Board of Robert Wood Johnson University Hospital Somerset (IRB17-16; January 4, 2018) and Rutgers Biomedical and Health Sciences (Pro20170001181; August 31, 2017).

\section{Author Contributions}

All authors made substantial contributions to conception and design, acquisition of data, or analysis and interpretation of data; took part in drafting the article or revising it critically for important intellectual content; agreed to submit to the current journal; gave final approval of the version to be published; and agree to be accountable for all aspects of the work.

\section{Disclosure}

This research received no external funding. Saira Chaudhry is an employee of Merck. The remaining authors report no conflicts of interest in this work. 


\section{References}

1. Hales CM, Carroll MD, Fryar CD, Ogden CL. Prevalence of obesity and severe obesity among adults: United States, 2017-2018. NCHS Data Brief. 2020;360:1-8.

2. Wang Y, Beydoun MA, Min J, Xue H, Kaminsky LA, Cheskin LJ. Has the prevalence of overweight, obesity and central obesity levelled off in the United States? Trends, patterns, disparities, and future projections for the obesity epidemic. Int J Epidemiol. 2020;49 (3):810-823. doi:10.1093/ije/dyz273

3. Ward ZJ, Bleich SN, Cradock AL, et al. Projected U.S. state-level prevalence of adult obesity and severe obesity. $N$ Engl J Med. 2019;381:2440-2450. doi:10.1056/NEJMsa1909301

4. Falagas M, Kompoti M. Obesity and infection. Lancet Infect Dis. 2006;6:438-446. doi:10.1016/S1473-3099(06)70523-0

5. Huttunen R, Syrjanen J. Obesity and the risk and outcome of infection. Int J Obes. 2013;37:333-340. doi:10.1038/ijo.2012.62

6. Bertakis KD, Azari R. Obesity and the use of health care services. Obes Res. 2005;13:372-379. doi:10.1038/oby.2005.49

7. Huttunen R, Laine J, Lumio J, Vuento R, Syrjänen J. Obesity and smoking are factors associated with poor prognosis in patients with bacteraemia. BMC Infect Dis. 2007;7:13. doi:10.1186/1471-2334-7-13

8. Bochicchio GV, Joshi M, Bochicchio K, Tracy K, Scalea TM. A time-dependent analysis of intensive care unit pneumonia in trauma patients. $J$ Trauma. 2004;56:296-301. doi:10.1097/01. TA.0000109857.22312.DF

9. Falagas ME, Athanasoulia AP, Peppas G, Karageorgopoulos DE. Effect of body mass index on the outcome of infections: a systematic review. Obes Rev. 2009;10:280-289.

10. Longo C, Bartlett G, Macgibbon B, et al. The effect of obesity on antibiotic treatment failure: a historical cohort study. Pharmacoepidemiol Drug Saf. 2013;22:970-976. doi:10.1002/pds.3461

11. Tucker CE, Lockwood AM, Nguyen NH. Antibiotic dosing in obesity: the search for optimum dosing strategies. Clin Obes. 2014;4:287-295. doi:10.1111/cob.12076

12. Janson B, Thursky K. Dosing of antibiotics in obesity. Curr Opin Infect Dis. 2012;25:634-649. doi:10.1097/QCO.0b013e328359a4c1

13. Narayanan N, Adams CD, Kubiak DW, et al. Evaluation of treatment options for methicillin-resistant Staphylococcus aureus infections in the obese patient. Infect Drug Resist. 2019;12:877-891. doi:10.2147/IDR. S196264
14. Sarmiento MRA, de Paula TO, Borges FM, et al. Obesity, xenobiotic intake and antimicrobial-resistance genes in the human gastrointestinal tract: a comparative study of eutrophic, overweight and obese individuals. Genes. 2019;10:349. doi:10.3390/genes10050349

15. Roe JL, Fuentes JM, Mullins ME. Underdosing of common antibiotics for obese patients in the ED. Am $J$ Emerg Med. 2012;30:1212-1214. doi:10.1016/j.ajem.2011.05.027

16. Roberts JA, Kruger P, Paterson DL, Lipman J. Antibiotic resistancewhat's dosing got to do with it? Crit Care Med. 2008;36:2433-2440. doi:10.1097/CCM.0b013e318180fe62

17. Garg A, Garg J, Kumar S, Bhattacharya A, Agarwal S, Upadhyay GC. Molecular epidemiology \& therapeutic options of carbapenem-resistant Gram-negative bacteria. Indian $J$ Med Res. 2019;149:285-289. doi:10.4103/ijmr.IJMR_36_18

18. Tacconelli E, Carrara E, Savoldi A, et al. Discovery, research, and development of new antibiotics: the WHO priority list of antibiotic-resistant bacteria and tuberculosis. Lancet Infect Dis. 2018;18:318-327. doi:10.1016/S1473-3099(17)30753-3

19. Centers for Disease Control and Prevention (CDC). Antibiotic Resistance Threats in the United States, 2019. Atlanta, GA: U.S. Department of Health and Human Services, CDC; 2019.

20. Thaden JT, Fowler VG, Sexton DJ, Anderson DJ. Increasing incidence of extended-spectrum $\beta$-lactamase-producing Escherichia coli in community hospitals throughout the Southeastern United States. Infect Control Hosp Epidemiol. 2016;37:49-54. doi:10.1017/ice.2015.239

21. Centers for Disease Control and Prevention (CDC). Vital signs: carbapenem-resistant Enterobacteriaceae. MMWR Morb Mortal Wkly Rep. 2013;62:165.

22. Clinical and Laboratory Standards Institute. Performance Standards for Antimicrobial Susceptibility Testing. 26th ed. CLSI supplement M100S. Wayne, PA: Clinical and Laboratory Standards Institute; 2016.

23. Vallianou N, Dalamaga M, Stratigou T, et al. Do antibiotics cause obesity through long-term alterations in the gut microbiome? A review of current evidence. Curr Obes Rep. 2021:1-19. doi:10.1007/s13679-021-00438-w

24. Anhê FF, Jensen BAH, Varin TV, et al. Type 2 diabetes influences bacterial tissue compartmentalisation in human obesity. Nat Metab. 2020;2(3):233-242. doi:10.1038/s42255-020-0178-9

25. Massier L, Chakaroun R, Tabei S, et al. Adipose tissue derived bacteria are associated with inflammation in obesity and type 2 diabetes. Gut. 2020;69(10):1796-1806. doi:10.1136/gutjnl-2019320118
Infection and Drug Resistance

\section{Publish your work in this journal}

Infection and Drug Resistance is an international, peer-reviewed openaccess journal that focuses on the optimal treatment of infection (bacterial, fungal and viral) and the development and institution of preventive strategies to minimize the development and spread of resistance. The journal is specifically concerned with the epidemiology of antibiotic resistance and the mechanisms of resistance development and diffusion in both hospitals and the community. The manuscript management system is completely online and includes a very quick and fair peerreview system, which is all easy to use. Visit http://www.dovepress.com/ testimonials.php to read real quotes from published authors. 